\title{
On a Method of Studying Displacement.
}

By R. F. Muirhead, MA., B.Sc.

Let a rigid body $F$ suffer a displacement to a new position, where it will be denoted by $F^{\prime}$

Then any point $A$ belonging to $F$ will take up a position $A^{\prime}$ in $F^{\prime}$. Let $B$ be the point of $F$ which coincides with $A^{\prime}$. Then corresponding to $B$ in $F$ there will be a point $B^{\prime}$ in $F^{\prime}$. Let this again coincide with $C$ in $F$, and so on. We have thus a sequence or chain of points whose relation may be thus indicated

\section{A B C DEFG .... $A^{\prime} B^{\prime} C^{\prime} D^{\prime} \mathbf{E}^{\prime} F^{\prime} G^{\prime} \ldots \ldots$}

That is to say, the figure ABCDEFG . . . in the body $F$ assumes the position $A^{\prime} B^{\prime} C^{\prime} D^{\prime} E^{\prime} F^{\prime} G^{\prime} \ldots$ in $F^{\prime}$ and in such a manner that the figure BCDEFG . . . coincides with $A^{\prime} B^{\prime} C^{\prime} E^{\prime} F^{\prime} \ldots$

That the construction may actually exist it would be necessary, in general, that the body $F$ should be indefinitely extended on all sides.

The construction just given forms the basis of the method of studying displacement which $I$ wish to put forward here.

It is at once obvious that $A B=B C$, for $A^{\prime} B^{\prime}$, which is merely $A B$ displaced, coincides with $B C$. Similarly $B C=C D=D E=$ etc.

Also the angles ABC, BCD, CDE, etc., are all equal for a similar reason, and the dihedral angles between each successive pair of the planes $A B C, B C D, C D E$, etc., are all equal. In fact the figure $\triangle B C D E F \ldots$ is a sort of regular rectilinear helix, which may degenerate, if the angles between the successive planes are zero, into a regular plane polygon, which will ultimately be closed or not, according as the angle $\mathbf{A B C}$ is or is not commensurable with a right angle.

Further, the figure may degenerate, if $\mathrm{ABC}=180^{\circ}$, into a series of equidistant collinear points.

The construction just explained applies equally to displacements in spaces of one, two, three, or more dimensions.

It enables us as it were to produce a displacement indefinitely, and may therefore be called the method of displacement-production; 
and the resulting figure may be called a displacement-sequence or displacement-chain. Any part of it is congruent with any other part of equal extent. Thus in solid space, the tetrahedron ABCD is congruent with the tetrahedron $B C D E$, and with the tetrahedron DEFG.

In unidimensional displacement, the sequence $\mathrm{AB}$ determines the displacement completely, in two dimensional motion the sequence $A B C$ does so ; in three-dimensionsl, the sequence $A B C D$, and so on.

The case of one-dimensional displacement, as of a line-body displaced along itself, is so simple as to require no special study.

\section{Two-dimengionat Digplacemrat.}

Take the case of motion of a plane body in a plane. Let ABCD be a displacement-chain.

Bisect the angles $\mathrm{ABC}, \mathrm{BCD}$ by lines $\mathrm{BO}, \mathrm{CO}$, meeting in 0 . Then

$$
\begin{gathered}
\angle \mathrm{OBC}=\frac{1}{2} \angle \mathrm{ABC}=\frac{1}{2} \angle \mathrm{BCD}=\angle \mathrm{BCO} \\
\therefore \quad \mathrm{BO}=\mathrm{CO} .
\end{gathered}
$$

But when B is displaced to C, the bisector BO comes to CO. Hence $O$ coincides with itself after displacement. Thus in any plane displacement there is one point that remains unmoved.

Corollary. Every plane displacement is equivalent to a rotation about a fixed point, the angle of rotation being the supplement of $\angle$ ABC.

Another Construction. Let $\mathrm{M}$ and $\mathrm{N}$ be the mid-points of $\mathrm{AB}$ and $\mathrm{BC}$, so that $\mathrm{MN}$ is a sequence.

Let $\mathrm{MO} \perp^{r}$ to $\mathrm{AB}$ and $\mathrm{NO} \perp^{r}$ to $\mathrm{BC}$ be drawn, meeting in 0 . Join OB.

In the triangles $O B M, O B N$, we have $M B=B N$ and $O B$ common, and $\angle \mathrm{OMB}=\angle \mathrm{ONB}=90^{\circ}$.

$$
\text { Hence } \quad O M=O N \text {. }
$$

But the line MO is displaced into NO. Hence, as before, 0 remains unmoved, and the fundamental theorem of plane displacement is proved.

As examples of other simple applications of displacementproduction in two dimensions, consider the following.

(1) If $\mathrm{AB}, \mathrm{BC}$ are collinear, the same is tive of the sequence of every other point in the body. Let $\alpha \beta \gamma$ be such another sequence. 
Join $A a, B \beta$. Then the figure $\mathrm{BA} \alpha$ displaces into $\mathrm{CB} \beta$

$$
\therefore \quad \angle \mathrm{BA} a=\angle \mathrm{CB} \beta \text {. }
$$

And $A \alpha=B \beta$, since the one displaces into the other.

Thus $A a$ is equal and parallel to $B \beta$

$\therefore \quad a \beta ", ", \quad " A B$ Similarly $\beta \gamma ", ", \quad ", \quad$,

$$
\therefore \alpha \beta \gamma \text { are collinear. }
$$

We see from this that in such a displacement, every point has a displacement equal and parallel to that of $\mathbf{A}$.

(2) If any two points $A$ and $a$ move through equal and parallel distances $\mathrm{AB}$ and $\alpha \beta$, in the same sense, so do all the others.

Let $a^{\prime} \beta^{\prime}$ be any other sequence.

Then $A \alpha \beta B$ is a parallelogram, $\therefore A \alpha$ is $\|$ to $B \beta$.

Again $\quad \angle A \alpha a^{\prime}=\angle B \beta \beta^{\prime}$.

$\therefore \alpha a^{\prime}$ and $\beta \beta^{\prime}$ are parallel ; and they are equal :

Hence $\quad a^{\prime} \beta^{\prime}$ is $=$ and $\|$ to $a \beta$.

(3) If any line remains parallel to itself, so do all lines that can be drawn in the body.

Let $A a$ be parallel to its displaced position $B \beta$ : the two are obviously equal. Hence $A \alpha \beta B$ is a parallelogram. Hence as in (2) every other point-displacement is equal and parallel to $\mathrm{AB}$ and $\alpha \beta$.

\section{Three-dimensional Displacement.}

To establish by this method the fundamental theorem of displacement in three dimensions, the following Lemma in Solid Geometry is required, of which an elementary proof is given later.

Lemma : Let BCYX be a tetrahedron such that

$$
\angle \mathrm{XBC}=\angle \mathrm{BCY} \text { and } \angle \mathrm{CYX}=\angle \mathbf{Y X B} \text {, }
$$

then it follows that $\mathrm{CY}=\mathrm{BX}$, also $\mathrm{CX}=\mathrm{BY}$, also the dihedral angles at equal edges are equal.

Proposition : In every displacement of a body in solid space, there is one straight line of the body which is displaced along itself.

Let $\mathrm{ABCDE}$ be a displacement-chain, and let $\mathrm{BX}, \mathrm{CY}, \mathrm{DZ}$ be 
the interior bisectors of the plane angles $A B C, B C D, C D E$; and let $\mathrm{XY}$ be the shortest distance between $\mathrm{BX}$ and $\mathrm{CY}$, i.e., the line which is perpendicular to each of them.

We have $\angle \mathrm{XBC}=\frac{1}{2} \angle \mathrm{ABC}=\frac{1}{2} \angle \mathrm{BCD}=\angle \mathrm{BCY}$

Hence by the Lemma, $\quad B X=C Y$.

Hence $X Y$ is a sequence; and the next point in this sequence is on $\mathrm{DZ}$ : let $Z$ be that point; then $\mathrm{YZ}$ is the shortest distance between $C Y$ and $D Z$.

We shall show that $X, Y, Z$ are collinear. Since the angles CYZ and CYX are right, it only remains to show that they are in the same plane.

Now by the Lemma the dihedral angles at the edges $\mathrm{XB}$ and $\mathrm{YC}$ are equal, in the tetrahedron $\mathrm{XBCY}$; and the dihedral angle at $\mathrm{CY}$ in the tetrahedron $\mathrm{YCDZ}$ being congruent with the former, is equal to the latter.

Thus the planes $\mathrm{XYC}$ and $\mathrm{ZYC}$ make equal angles with the plane BCDY, and are on opposite sides of it. Hence they are parts of the same plane.

Hence $\mathrm{XY}$ and $\mathrm{YZ}$ are collinear.

Thus in the given displacement the line XYZ is displaced along itself.

Corollary. The displacement is equivalent to a screw motion, the pitch of the screw having the same ratio to XY that $360^{\circ}$ has to the angle between the planes BXY and XYC.

Another proof, perhaps more intuitive but less complete, is as follows :

If we draw, from some fixed point, lines in the same direction as $\mathrm{AB}, \mathrm{BC}, \mathrm{CD}$, etc., respectively, the angles between those lines, taken successively in pairs, are all equal, and also the angles between the planes containing successive pairs. Thus we get a figure which bears the same resemblance to a circular cone that a regular (closed or unclosed) polygon bears to a circle. Obviously a line equally inclined to three of these lines is equally inclined to all.

Now the shortest distance $\mathrm{XY}$ of the previous figure is clearly equally inclined to $\mathrm{AB}$ and $\mathrm{BO}$, since it is perpendicular to the bisector BX. Similarly it is equally inclined to BC and CD. Thus 
it is equally inclined to three links of the chain, and, therefore, to all. Hence it is in the same direction as the shortest distance between $\mathrm{CY}$ and $\mathrm{DZ}$.

Again, the two successive shortest distances are coterminous in $\mathrm{Y}$, since by symmetry $\mathrm{BX}=\mathrm{CY}$. Other simple applications to three dimensions are as follows:-

(1) If $A B C$ are collinear, then either $A B C$ is the axis of the screw, or else the displacement is a pure translation.

(2) If $\mathrm{ABC}$ are collinear and $\alpha \beta \equiv \mathrm{AB}$, then there is translation only (using the symbol $\equiv$ to denote that the two lines are $=, \|$ and in the same sense).

For if we take any third sequence $a^{\prime} \beta^{\prime}$, the tetrahedra $\alpha^{\prime} \mathrm{AB} a$ and $\beta^{\prime} \mathrm{BC} \beta$ being in sequence, are congruent; and their bases $\alpha A B$ and $\beta B C$ being in the same plane, we have clearly $a^{\prime} \beta^{\prime} \equiv A B \equiv \alpha \beta$.

(3) If three sequences, $\mathrm{AB}, \mathrm{A}^{\prime} \mathrm{B}^{\prime}, \alpha \beta$, are such that $A B \equiv A^{\prime} B^{\prime} \equiv \alpha \beta$, then all sequences of two points are so.

For if $\alpha^{\prime} \beta^{\prime}$ be any other sequent pair, the tetrahedra $\mathrm{AA}^{\prime} \alpha a^{\prime}$ and $\mathrm{BB}^{\prime} \beta \beta^{\prime}$ being congruent and on homothetic bases $\mathrm{AA}^{\prime} a$ and $\mathrm{BB}^{\prime} \beta, \therefore \alpha^{\prime} \beta^{\prime} \equiv \alpha \beta \equiv$ etc.

This fails only when $\mathrm{AA}^{\prime} a$ are collinear, in which case the second datum is not independent of the first, so that the case reduces to (4).

(4) If there are two sequences $A B$ and $\alpha \beta$ such that $A B \equiv \alpha \beta$, then the axis of the screw must be parallel to $A a$, or else the displacement is a pure translation.

For if we take any other line in the body $\equiv A a$, say $A^{\prime} a^{\prime}$, then $A a a^{\prime} A^{\prime}$ and $B \beta \beta^{\prime} B^{\prime}$ are parallelograms, as also $A a \beta B$. Hence

$$
A^{\prime} \alpha^{\prime} \equiv A \alpha \equiv B \beta \equiv B^{\prime} \beta^{\prime} \text {. }
$$

Thus any line $\|$ to $A \alpha$ remains so. Hence $A \alpha$ is parallel to the sorew-axis, if there is one.

(5) If $A B C D$ are coplanar but not collinear, two cases may occur (i.) the plane $A B C$ of the body may be facing the same way in its displaced position BCD, or (ii.) it may have been turned over; i.e., the angle between successive planes in sequence may be $0^{\circ}$ or $180^{\circ}$. In the former case, all points in the plane $A B C$ evidently remain in it, and therefore (as in uniplanar displacements) one 
point in the plane is unmoved, and the displacement is a rotation about an axis through this point perpendicular to the plane ABC.

In the latter case, the sequence $\mathrm{ABCD}$. . . forms a zig-zag figure, consisting of equal distances which are parallel alternately, so that $\mathrm{AB}, \mathrm{CD}, \mathrm{EF}$, etc., are parallel to one another, and also BC, DE, etc., are parallel to one another. It follows that ACEG . . a are collinear and parallel to BDFH .... This indicates that the axis of the screw is the parallel line midway between $\mathrm{ACE}$ and $\mathrm{BDF}$, and that the rotation about this axis is $180^{\circ}$.

If we restrict our attention to the plane body $A B C D$ in this case, we see that it corresponds to inversion in a plane, and we can at once deduce several useful propositions with respect to inversion of plane figures: e.g., that when a plane figure is inverted, there is one direction which remains unaltered, and another at right angles to it which is exactly reversed: also that one line in the former direction slides along itself, but all those in the latter direction are displaced by the same amount parallel to themselves.

It may be remarked that in general an inversion in any space is equivalent to a special case of displacement in the next higher space.

Let us now apply to three-dimensional displacement a construction similar to the second construction in two-dinensional displacement. Let $\mathrm{P}, \mathrm{Q}, \mathrm{R}, \mathrm{S}$ be the axial planes of the pairs of points $A B, B C, C D, D E$ respectively, $A B C D E$ being a sequence, and the axial plane being the locus of points equidistant from each point of the pair in question.

$P, Q$, and $R$ intersect in a point $O$. Then $O$ is equidistant from $A, B, C, D$.

Similarly $Q, R, S$ intersect in a point $O^{\prime}$ equidistant from B, C, D, E.

Now compare the two tetahedra OBCD and $O^{\prime} B C D$. Obviously their corresponding edges are equal, each to each. Are they then congruent? If so it would follow that $O$ and $O^{\prime}$ were coincident, and therefore that in three-dimensional displacement there is a point that remains unmoved, which is not so.

The explanation is that $\mathrm{OBCD}$ and $\mathrm{O}^{\prime} \mathrm{BCD}$ are symmetric, not congruent, i.e., they bear to one another the same relationship that a body bears to its image in a plane mirror. We may say that one is the reflex, or reverse of the other. To prove this we may note 
that $O$ and $O^{\prime}$ are the centres of the circumspheres of $A B C D$ and of $\mathrm{BCDE}$ respectively. If we can show that $A$ and $E$ are on opposite sides of the plane $B C D$, it will follow that $O$ and $O^{\prime}$ are also on opposite sides of that plane.

We note that the tetrahedron EBCD is congruent with DABC, the corresponding points being in the order stated. Now if the circuit $A B C$, when looked at from $D$, is clockwise, we see that the circuit $\mathrm{BCD}$, looked at from $\mathrm{A}$, will be counter-clockwise, and vice versa. But $\mathrm{EBCD}$ is congruent with $\mathrm{DABC}$, and therefore the circuit $B C D$, seen from $E$, is in the same sense as the circuit $A B C$, seen from $D$, therefore in the sense opposite to that of $B C D$ seen from $A$. Hence $A$ and $E$ must be on opposite sides of the plane BCD. Thus the explanation is complete.

Noting that in one-dimensional displacement, and in threedimensional displacement there is a line which coincides with itself, but no self-coincident point, while in two-dimensional motion, the reverse is the case, we might suspect that for displacement in space of even dimensions there is always one point fixed, but no selfcoincident line, while the reverse is the case for displacement in space of odd dimensions.

This is true, and it can further be shown that in $n$-dimensional displacement that is always a fat $(\overline{n-2})$-fold space which moves in itself, from which it follows that there is an $\overline{n-4}$-fold flat space in that which moves in itself, and so on, down to a point in the case of $n$ even, or a line in the case of $n$ odd.

From this we deduce the remarkable result that the most general displacement in $2 n$-fold space is equivalent to $n$ rotations, and that in $(2 n+1)$-fold space the most general displacement is equivalent to $n$ rotations together with a translation.

For, calling a flat $n$-fold space $S^{n}$ for brevity, we may reason thus:-in the case of displacement in $\mathbf{S}^{2 n}$ we begin by keeping fixed the $\mathrm{S}^{2 n-2}$ which is perpendicular to the self-coincident plane of the body and meets it in the self-coincident point. A rotation about this $\mathrm{S}^{2 n-2}$ will bring all points of the said plane into their tinal positions. Next keep fixed the $S^{2 n-2}$ which is perpendicular to the self-coincident $S^{4}$ and meets it in the plane before mentioned. This gives rise to a rotation which brings every point of the $\mathrm{S}^{4}$ into its final position. Proceeding thus we finally reach the displaced position of the body by means of $n$ rotations. 
In the case of displacement in a $\mathbf{S}^{2 n+1}$ we may begin by a displacement along the self-coincident line, bringing its points into their final positions.

But the proof, or even the complete statement of these matters would unduly weight this paper, which aims rather at explaining the method than at exhausting its applications ; so for the present I shall content myself by giving the elementary proof of the statement that in a space of even dimensions there is one point that does not move.

It is obvious that an extension of the second construction given in two-dimensional displacement goes far towards this proof. We must in $n$-dimensional displacement take a chain of $n+2$ points $A, B, C \ldots H, K, I_{4}$, and construct the axial $\mathbf{S}^{n-1}$ for each successive pair. Let these axial $\mathbf{S}^{n-1} \mathbf{g}$ be $\mathrm{P}, \mathrm{Q}, \mathbf{R} \ldots \mathrm{T}, \mathrm{U}$. They are $n+1$ in number. The first $n$ of them intersect in a point $O$, and the last $n$ of them in a point $O^{\prime}$. As in the case of three dimensions, $O$ and $O^{\prime}$ will be coincident or not according as $A$ and $L$ lie on the same or on opposite sides of the $S^{n-1}$ determined by BC . . HK. This again will depend on whether the figure $B O$. . BK has the same sense as seen from $I$ and from $A$ or not. But since KABC . . . H and LBC . . . HK are congruent, $A B C \ldots H$ seen from $K$ and $B C$. . HK seen from $L$ have the same sense. Thus $O$ and $O^{\prime}$ will be coincident or not according as $B C$. . . HK seen from $A$ and $A B C \ldots H$ seen from $K$ have the same sense or not, or finally according as the figure $\mathrm{ABC}$. . H HK, which is a simplissimum in $n$ dimensions, has not or has its sense altered by changing the order of its points to $\mathrm{KABC}$. . H. H.

This implies a conception for $n$ dimensions equivalent to that of the distinction between a positive and a negative circuit in a plane. If we form such a conception, we find that the interchange of two adjacent letters changes the sense of the figure. Hence an odd number of such interchanges will result in a figure of opposite sense, and an even number in one of the same sense. Now in the "circuit" $\mathrm{ABC}$... HK there are $n+1$ letters, so that $n$ interchanges are required to make the change to $\mathrm{KABC} \ldots \mathrm{H}$. Hence the sense of the figure is altered or not according as $n$ is odd or even.

Hence if $n$ be even $\mathrm{O}$ and $\mathrm{O}^{\prime}$ coincide, that is there is one point unmored in the general displacement.

I may mention that in the Annales de l'École Polytechnique de 
Delft, t. VII. (1891), pp. 139-158, Mr P. H. Schoute has a paper entitled "Le Déplacement le plus général dans l'espace à $n$ Dimensions," in which he demonstrates the resolution above stated in connection with the general theory of projectivity in $S^{n}$ by the aid of homogeneous coordinates. In this paper, which is a very interesting and elegant one, he refers to an earlier paper on the same subject by Rahusen, t. IV., p. 104, of the same Annales, and also to the treatise by G. Veronese entitled "Fondamenti di Geometria."

\section{Appendix: Elementary Geonetry of the Isosceles Skew Trapezium.}

Definition: Let a skew quadrilateral $\mathrm{ABCD}$ having $\angle \mathrm{A}=\angle \mathrm{B}$ and $\angle \mathrm{O}=\angle \mathrm{D}$ be called an Isosceles Skew Trapezium.

Prop. 1. In such an isosceles skew trapezium $\mathrm{AC}=\mathrm{BD}$ and $\mathrm{AD}=\mathrm{BC}$.

Construction: Draw $\mathrm{AE} \equiv \mathrm{DC}$, also $\mathrm{BF} \equiv \mathrm{CD}$ (using the symbol $\equiv$ to mean equal and in the same direction).

Join CE, EB, AF, FD. Then AECDFB is a triangular prism.

Now the trihedral solid angle $A(B D E)$ is congruent with the solid angle $B(A C F)$, for

$$
\begin{aligned}
& \angle \mathrm{EAD}=180^{\circ}-\angle \mathrm{ADC}=180^{\circ}-\angle \mathrm{DCB}=\angle \mathrm{CBF} \\
& \angle \mathrm{EAB}=\angle \mathrm{FBA} \\
& \angle \mathrm{DAB}=\angle \mathrm{CBA}
\end{aligned}
$$

whence the congruity follows by Bk. XI. Additional Prop. 1 of the Pitt Press Euclid.

$\therefore$ dihedral angle $\mathrm{E} \overline{\mathrm{ABD}}(\overline{\mathrm{AB}}$ being the edge $)=$ dihedral angle $\mathrm{F} \overline{\mathrm{BA}} \mathrm{C}$ $\therefore$ dihedral $\mathrm{D} \overline{\mathrm{ABF}}=$ dihedral $\mathrm{C} \overline{\mathrm{ABE}}$

Again the trihedrals $\mathrm{A}(\mathrm{DBF})$ and $\mathrm{B}(\mathrm{CAE})$ have

$$
\begin{aligned}
& \angle \mathrm{BAE}=\angle \mathrm{ABF} \\
& \angle \mathrm{DAB}=\angle \mathrm{CBA}
\end{aligned}
$$

dihedral $\mathrm{DA} \overline{\mathrm{AB}} \mathrm{F}=$ dihedral $\mathrm{C} \overline{\mathrm{AB}} \mathrm{E}$

Hence by Add. Prop. 2, Bk. XI. of the Pitt Press Euclid they are congruent.

$$
\begin{aligned}
\therefore \angle \mathrm{DAF} & =\angle \mathrm{CBE} . \\
& =\angle \mathrm{DFA}
\end{aligned}
$$

$\therefore \mathrm{DA}=\mathrm{DF}=\mathrm{CB}=\mathrm{CE}$.

But the $\triangle \mathrm{s} \mathrm{DAB}, \mathrm{CBA}$ are congruent by Euc. I., 4. $\therefore \mathrm{CA}=\mathrm{DB}$. 
Prop. 2. Converse of Prop. I. If $\angle \mathrm{C}=\angle \mathrm{D}$ and $\mathrm{CB}=\mathrm{DA}$, then $A B C D$ is an isosceles skew trapezium.

For the $\triangle \mathrm{s} \mathrm{DCB}, \mathrm{CDA}$ are congruent by Euc. I., 4 .

$$
\therefore \quad, \quad \text { ABC, BAD , " " , I., } 8 \text {. }
$$

Q. E. D.

Prop. 3. Another converse of Prop. I. If $\mathrm{CB}=\mathrm{AD}$ and $\mathrm{CA}=\mathrm{BD}$, the same result follows easily by Euc. I., 8 .

Prop. 4. In an isosceles skew trapezium $\mathrm{ABCD}$ as above, if $\mathrm{H}, \mathrm{K}$ be the mid points of $\mathrm{CD}, \mathrm{AB}$, then $\mathrm{HK}$ is perpendicular both to $\mathrm{AB}$ and to $\mathrm{CD}$.

The triangles $\mathrm{BCH}, \mathrm{ADH}$ are congruent, by Euc. I., 4 .

$\therefore \mathrm{HA}=\mathrm{HB} . \quad \therefore \angle \mathrm{HKA}=\angle \mathrm{HKB}$.

$\therefore \mathrm{HK}$ is $\perp^{r}$ to $\mathrm{AB}$.

Similarly $H K$ is $\perp^{r}$ to CD.

Thus HK is the shortest distance between $A B$ and $C D$.

Prop. 5. If $\mathrm{XY}$ be the shortest distance from $\mathrm{AD}$ to $\mathrm{CB}$, then $A$ and $B$ are equally distant from $X$ and from $Y$ respectively; and so also are $C$ and $D$.

This is a corollary of Prop. I.

Conversely, an isosceles skew trapezium may be constructed in two ways, thus :-

(1) Take two pairs of points $(A, B),(D, C)$ on two lines, BCX, $\mathrm{ADY}$ in space, whose shortest distance is $\mathrm{XY}$, such that $\mathrm{CY}=\mathrm{DX}$ and $B Y=A X$. Then $A B C D$ is an isosceles skew trapezium having $\angle \mathrm{C}=\angle \mathrm{D}$ and $\angle \mathrm{A}=\angle \mathrm{B}$.

(2) Take two lines $\mathrm{AB}, \mathrm{CD}$ in space and let $\mathrm{HK}$ be their shortest distance, $\mathrm{C}$ and $\mathrm{D}$ being equally distant from $\mathrm{H}, \mathrm{A}$ and $\mathrm{B}$ being equally distant from $\mathrm{K}$. Then $\mathrm{ABCD}$ is an isosceles skew trapezium having $\angle \mathrm{A}=\angle \mathrm{B}$ and $\angle \mathrm{C}=\angle \mathrm{D}$.

Figure (34) shows the isosceles skew trapezium $A B C D$ in relation to its shortest distances $\mathrm{XY}$ and HK.

It is obvious that $\mathrm{HK}$ is the locus of the mid points of lines joining points on $X A, Y B$, which are equally distant from $X$ and $Y$ respectively: also that the figure is symmetrical about the line LHK.

On Superposition by the Aid of Dissection.

By R. F. Muirhead, M.A., B.Sc. 